\title{
Erratum to: Adult de novo lumbar scoliosis. Posterior instrumented fusion with Smith-Peterson osteotomy, decompression and management of postoperative infection
}

Claudio Lamartina - Maria Petruzzi

Published online: 19 October 2011

(C) Springer-Verlag 2011

Erratum to: Eur Spine J (2011) 20:1580-1581

DOI 10.1007/s00586-011-1941-0

The following affiliation was omitted:

I.R.C.C.S Istituto Ortopedico Galeazzi, Milan, Italy.

e-mail: c.lamartina@chirurgiavertebrale.net.

The online version of the original article can be found under doi:10.1007/s00586-011-1941-0.

C. Lamartina $(\bowtie) \cdot$ M. Petruzzi

I.R.C.C.S Istituto Ortopedico Galeazzi, Milan, Italy

e-mail: c.lamartina@chirurgiavertebrale.net 